\title{
A Multi-objective Evolutionary Algorithm for Seamless Steel Tube Order-grouping Problem
}

\author{
Shen Zhao ${ }^{1, \text { a }}$, Zhang Wenxin ${ }^{1, \text { a }}$, Liu Jie ${ }^{1, \text { a }}$ \\ ${ }^{1}$ Donlinks School of Economics and Management, University of Science and Technology Beijing, \\ Beijing, China; \\ ${ }^{a}$ Engineering Research Center of MES Technology for Iron \& Steel Production, Ministry of Education, \\ China \\ mimashen6357@sina.com
}

Keywords: order-grouping problem, multi-objective optimization, genetic algorithm, seamless steel tube.

\begin{abstract}
The order-grouping problem of Hot Rolling Seamless Steel Tube is studied in this paper. A multi-objective optimization model minimizing overall delivery date and address difference is established, then a multi-objective evolutionary algorithm with elite-strategy and crowding distance computing is designed to solve this problem. The experiment indicates that the algorithm can solve this problem effectively and is useful for practical production.
\end{abstract}

\section{Introduction}

Seamless steel tube is useful, but working out its production plan is very difficult, because it has complex production processes and a variety of products. Experts and scientists have done some researches and got some results about order-grouping problem. Wang Haifeng [1] researched production plan problem basing on constraint satisfaction technique, and realized order-grouping of tube blank orders by means of grouping technology; Liu Guilin [2] and $\mathrm{Li} \mathrm{Hu}$ [3] built two optimization models for short and unfixed orders, considering production lot-sizing and tube sawing length and solved it by heuristic algorithms. Li Qingyu [4] addressed an order-grouping problem connecting order-grouping with production materials-grouping.

All these researches have solved this problem, but they emphasized process constraints instead of joining with delivery. However, an optimal distribution scheme will not only benefit schedule of production, but also reduce inventory and transporting costs. So, this paper has taken delivery optimization into consideration.

\section{Problem model}

The order-grouping problem can classify the orders into several rolling batches considering the attributes of the orders. In this paper, the hot rolling seamless steel tube order-grouping problem is considered basing on production and delivery optimization. As a result, the production and delivery costs are reduced.

\subsection{Assumptions}

Here are two assumptions in this paper: 1) This paper only considers the fixed length orders; 2) A large order can be disassembled into some smaller ones if its required length is over batch's max rolling length.

\subsection{Notations}

Parameters and variables are described as follows in this optimization model:

1) indexes and sets

$I$ : order set, $I=\{1,2, \ldots, n\} ; \quad i$ : order number, $i \in I$; 
$J$ : rolling batch set, $J=\{1,2, \cdots \cdots, m\} ; \quad j$ : rolling batch number, $j \in J$;

Matrix R represents the weight of distance. Every value in this matrix means the distance weight of the two corresponding distribution areas.

2) Parameters

$\left[d e_{i}, d l_{i}\right]:$ delivery time window, $i \in I$;

$r_{i i^{\prime} j}$ : The distance weight of delivery address between order $i$ and order $i^{\prime}$, its value is from matrix R;

$p_{i}$ : rolling length of order $i, i \in I ; \quad \phi_{i}$ : steel specification of order $i, i \in I ;$

$g_{i}$ : steel grade of order $i, i \in I ; \quad p_{\max }$ : maximum rolling length of a single batch;

3) Variables

$x_{i j}$ represents whether the order $i$ belongs to the batch $j$, if it is, $x_{i j}=1$, or $x_{i j}=0$.

\subsection{Problem model}

Basing on the assumptions and variables above, the multi-objective grouping optimization model is as follows:

$$
\begin{aligned}
\min f_{1} & =\sum_{i \in I} \sum_{i^{\prime} \in I} \sum_{j \in J} x_{i j} \cdot x_{i^{\prime} j}\left|\left(d e_{i}+d l_{i}\right) / 2-\left(d e_{i^{\prime}}+d l_{i^{\prime}}\right) / 2\right| / 2 \\
\min f_{2} & =\sum_{i} \sum_{i^{\prime}} \sum_{j} x_{i j} x_{i^{\prime} j} r_{i i^{\prime} j}
\end{aligned}
$$

s.t.

$\sum_{i \in I} x_{i j} \geq 1, j \in J$

$\sum_{j} x_{i j}=1, i \in I$

$x_{i j} \cdot \phi_{i}=x_{i^{\prime} j} \cdot \phi_{i^{\prime}}, i \in I, i^{\prime} \in I, j \in J$

$x_{i j} \cdot g_{i}=x_{i^{\prime} j} \cdot g_{i^{\prime}}, i \in I, i^{\prime} \in I, j \in J$

$\sum_{i \in I} x_{i j} \cdot p_{i} \leq p_{\max }, j \in J$

Objective Function (1) is to minimize the difference of total delivery date of all the rolling batches. Objective Function (2) is to minimize the difference of total distance weight of all the rolling batches. Constraint (3) represents each rolling batch must have at least one order. Constraint (4) represents that each order only belongs to one rolling batch. Constraint (5) and (6) represent that the required steel specification and grade of orders which is in the same rolling batch must be the same. Constraint (7) represents that total rolling length of a rolling batch must be under the $p_{\max }$

\section{Solving algorithm}

Order-grouping problem of seamless steel tube is NP-hard, and it is usually with multiple objectives and constraints. This paper give a multi-objective evolutionary algorithm to solve this problem.

\subsection{Encoding method}

Order grouping problem is a typical bin packing problem. Rolling batches represent the boxes and orders represent the objects. There are two kinds of encoding modes about bin packing problems: Bin-Based Representation (BBR) and Object-Based Representation (OBR).This paper applies BBR, 
which means that position of gene represents order, and the value of gene represents rolling batch that order belongs to.

For example, "0122" means that Order 1 belongs to Batch 0, and Order 2 belongs to Batch 1, Order 3 and Order 4 belong to Batch 2.

\subsection{Fitness function}

Fitness value of individuals which are in Pareto optimal solution set is 1. A individual, as $x$, which is in the same population $P(t)$ but not in Pareto optimal solution set, gets its fitness value by the following formula:

$$
f(x)=\frac{1}{1+\sqrt{\sum_{m=1}^{2}\left[f_{m}(x)-f_{m}(y)\right]^{2}}}
$$

$y$ is the individual which is in the Pareto optimal solution set and closest to $x$. Individuals are more likely to be selected if they are closer to Pareto optimal solution set.

\subsection{Crowding distance}

Pareto optimal solution set has its size M. Some individuals need to be eliminated when the individual number of Pareto set is over $\mathrm{M}$. its processes are as follows:

Step1: Sort objective value from min to max, and use formula

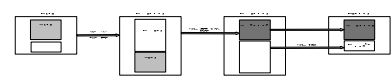
to calculate each individual's crowding distance about each subjective function $f_{m}$.

Step2: eliminate the individual who possesses the min crowding distance.

Step3: if the number of individuals of the Pareto optimal solution set is less than or equal to $\mathrm{M}$, end; or return to step1.

This doing can avoid the unlimited growth of better individuals, or the larger and larger size may affect the efficiency of algorithm and the genetic diversity.

\subsection{Detailed processes}

The multi-objective evolutionary algorithm generates a new population using elite strategy of Genetic Algorithm. Conduct crossover and mutation operations among the overall last generation $P(t)$, then constitute a new mid generation $P^{\prime}(t+1)$ with combining Pareto optimal solution set of the generation $P(t)$, and then conduct selection operations among the individuals of the $P^{\prime}(t+1)$ which is not in Pareto optimal solution set, finally $P^{\prime}(t+1)$ is as the new generation $P(t+1)$.The detailed steps of the algorithm are as follows:

Step1: Set the population size (PopSize), the size of Pareto optimal solution set (M), max number of iterations (NG), probability of crossover $\left({ }^{P c}\right)$ and probability of mutation $(P m)$;

Step2.1: produce orders randomly;

Step2.2: generate initial population and get the Pareto optimal solution set of initial population;

Step3.1: group orders to original order sets on condition that steel specification and grade must be same;

Step3.2: calculate the minimum number of rolling batches of each original order set by the formula $M=\left\lfloor\sum_{i=1}^{s} p_{i} / p_{\max }\right\rfloor$;

Step4: conduct genetic operation among population (Pop), and constitute a new mid population (m-Pop) with combining the current Pareto optimal solution set.

Step5.1: get the Pareto optimal solution set of this mid population

Step5.2: determine whether its scale is over M? yes, conduct next step ; no, turn to step 5.4; 
Step5.3: calculate crowding distance circularly of each individual, eliminate the individual who has minimum crowding distance, and then return step 5.2;

Step5.4: update and save the current Pareto optimal solution set;

Step6: calculate fitness value of every individual who is in m-Pop, and then eliminate the minimum one until population size is not over PopSize;

Step7; replace Pop with m-Pop and determine whether the iteration number if over NG. yes, conduct next step; no, turn to step4;

Step8: classify the best individual of Pop, decode, and output group batches result.

\section{Experiment results and analysis}

This algorithm is coded by C\#, using software Microsoft visual studio 2013, and run under OS Windows7-32bit. The algorithm parameters are set as follows: the sizes of order are 100,150,200; max number of iteration is 150; probability of crossover and mutation is 0.9 and 0.02 . Firstly, produce samples randomly; then, compute them with the algorithm for ten times and record objective value of each time and each iteration and algorithm running time; finally, get 10 times' average value of them. As shown in Table 1.

Table 1 Test Results

\begin{tabular}{cccc}
\hline Order Size & Objective & Objective2 & Running Time \\
\hline 100 & 4012 & 1715 & $15.8 \mathrm{~s}$ \\
\hline 150 & 7740 & 3111 & $26.3 \mathrm{~s}$ \\
\hline 200 & 10452 & 4266 & $43.5 \mathrm{~s}$ \\
\hline
\end{tabular}

As is shown in Table 1, this algorithm is comparatively stable. When the order size is 100 , the average computing time is $18.5 \mathrm{~s}$; when the order size is 150 , the average computing time is $26.3 \mathrm{~s}$; when the order size is 200 , the average computing time is $43.5 \mathrm{~s}$. Generally speaking, with the increasing of order size, the time of the algorithm will increase, but this algorithm can be completed within 50 s when the size of order is 200 . So that, the algorithm is efficient.

Orders whose size is 100 are produced randomly to testify the astringency of this algorithm further. Results as shown in Fig.1.

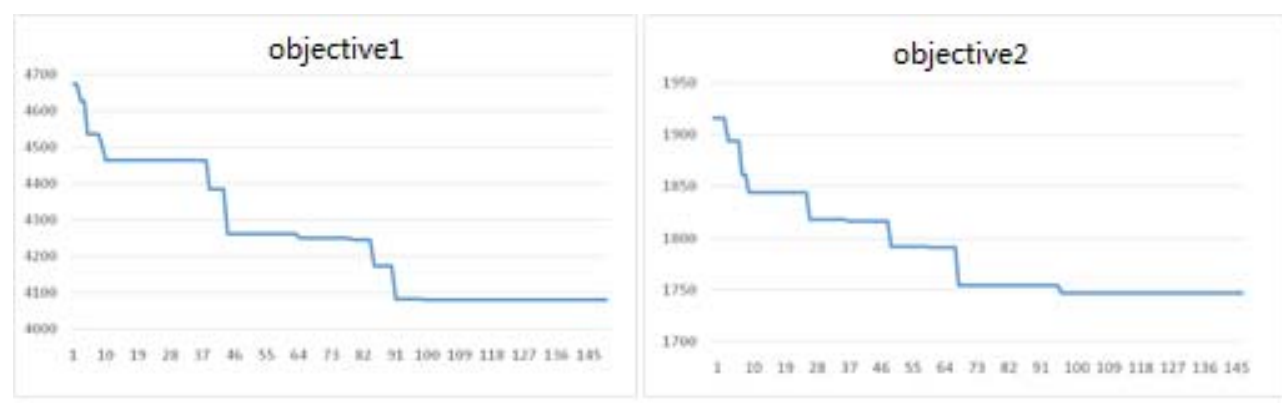

Fig. 1 Convergence of the algorithm.

From the Fig.1, we know that the value of both object functions declines at a relatively quick speed, because elite strategy gives privilege to better solutions to enter the next generation directly, which could accelerate the speed of convergence. What's more, values of the both objectives trend to be stable after 100 generations, illustrating that the algorithm has a good astringency.

\section{Conclusions}

The order grouping problem of hot rolling seamless steel tubes is of great practice and importance for Iron and steel enterprises' daily production management. This problem belongs to multi-objective optimization problem for its usually considering of more than one objectives and constraints. This paper aims at minimizing the difference of order delivery date and delivery address, then, constructs a multi-objective optimization model and designs a multi-objective evolutionary algorithm with elite 
strategy and crowding distances computing. After experiment with random samples, results manifest that the algorithm is effective.

\section{References}

[1] H. Wang, J. Peng, and Q. Zhang, "Hot rolling seamless steel tube production plan model based on constraint satisfaction and the algorithm," Chin. Mana. Info, vol. 15, pp. 43-45, November.2012.

[2] G. Liu, "Optimization algorithm of short and unfixed order-grouping in steel tube production," Bao. Gang. Tech, vol.2, pp. 42-44, 2007.

[3] H. Li and J. Huo, “Optimization algorithm of order-grouping in steel tube production,” Indu. Engi. Mana, vol 1, pp. 86-88, 2004.

[4] Q. Li, H. Zhu. "Research on supply chain customer order-grouping optimization technology of the Iron and Steel enterprise”. Industrial engineering and management, 2203(2):51-53.

[5] J. Tong, M. Zhao, "The multi-objective evolutionary algorithm which is effective to solve the Pareto optimal front”. Computer simulation, 2009, 26(6): 216-219.

[6] G. Xuan, R. Cheng. "Genetic algorithm and engineering optimization" .Beijing: Tsinghua University Press, 2004:48-54. 